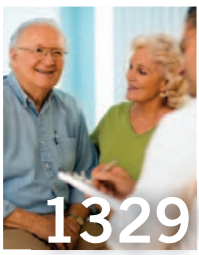

Senior sequence:

Geriatric genomics

aims to spur new

technologies

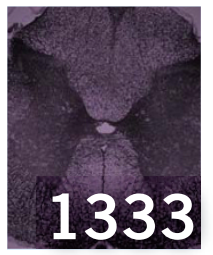

ALS not well:

Experts debate the

root cause of Lou

Gehrig's disease

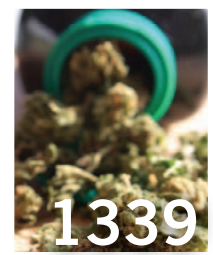

Gone to pot:

Research into

'legal highs' proves

chronically lacking

\title{
Preemptive genotyping trialed to prevent adverse drug reactions
}

\section{MONTREAL - In March 2010, the US Food} and Drug Administration (FDA) added a 'black box' warning to the drug clopidogrel, noting the link between certain genotypes and reduced drug efficacy. Despite the label change, however, only around one in every 200 physicians actually orders a genetic test when prescribing clopidogrel—an antiplatelet medicine marketed by Sanofi and Bristol-Myers Squibb as Plavix-according to data from Medco Health Solutions, a New Jersey-based pharmacy services provider.

The slow uptake of such testing is not due to some ingrained aversion to pharmacogenetic information, however. In work presented at the International Congress of Human Genetics here last month, Medco's senior director of personalized medicine Lon Castle found that $30 \%$ of cardiologists and $41 \%$ of primary care providers agreed to free genetic testing when contacted after having prescribed clopidogrel for their patients. Physicians "are willing to do it; they're eager to do it-they just need some more information to make themselves comfortable with it," Castle says.

But after-the-fact testing is already one step too late, says Dan Roden, assistant vicechancellor for personalized medicine at the Vanderbilt University Medical Center in Nashville, Tennessee. At the meeting here last month, Roden and his colleagues reported the results of a retrospective review of the records of nearly 53,000 regular users of the Vanderbilt health system. They looked at just six of the 99 drugs with FDA-sanctioned pharmacogenetic information, including clopidogrel, and showed that between 300 and 600 serious adverse drug reactions could have been avoided over the past five years had preemptive genotyping coupled to effective intervention strategies been in place.

With an eye to pharmacogenomically guided drug therapy, in September 2010 Vanderbilt launched PREDICT (short for Pharmacogenomic Resource for Enhanced Decisions in Care and Treatment), a systemwide effort to proactively link genetic information to the institution's extensive electronic health record system and then alert physicians through a pop-up notice if a patient's genetics predict any complications

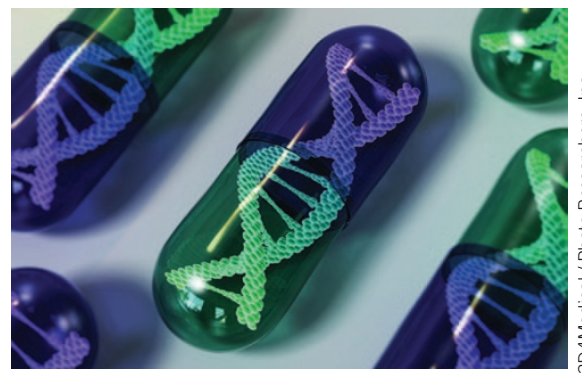

Drug response: Pharmacogenetics in the clinic.

with medicines they plan to prescribe. As a first step to broader implementation, the trial is starting out with clopidogrel response in people undergoing heart catheterizations-a standard procedure performed on more than a million Americans each year so that doctors can monitor their patients' coronary arteries for any signs of illness. To date, more than 3,000 people entering the 'cath lab' have also received a genetic test for DNA variants that have been linked to drug responses-information that then goes into their medical record.

John McPherson, an interventional cardiologist at Vanderbilt, recalls one such trial participant whose genetics predicted that he would be a poor metabolizer of clopidogrel, a compound that must be converted into its active form by a liver enzyme known as CYP2C19 to have a full effect. So McPherson prescribed this man Effient (prasugrel), another antiplatelet agent sold by Daiichi Sankyo and Eli Lilly that doesn't have the same limitations, although it does carry an increased risk of major bleeding. Six months on, the man is doing fine.

"If he wasn't part of this trial, most likely he would have been placed on clopidogrel because that's what we do, and who knows what would have happened to him," says McPherson. The PREDICT trial "has changed the way that I've treated these patients."

\section{Rebranding medicine}

As part of the US National Institutes of Health Pharmacogenomics Research Network (PGRN), similar programs involving clopidogrel are also underway or planned at Ohio State University, the Mayo Clinic and the Universities of Florida and Maryland.
"It's a different brand of science than any of us have done before," says PGRN member Alan Shuldiner, who is leading the Maryland clopidogrel trial as a well as a similar effort outside of the PGRN involving warfarin. "It's basically the science of how you implement a pharmacogenomic finding."

"Eventually, genetic information will be available on all patients, and part of making that a clinical reality is helping the health system know what to do with that information," says PGRN member Julie Johnson, a pharmacotherapy expert at the University of Florida in Gainesville.

Mary Relling, a clinical pharmacologist at St Jude Children's Research Hospital in Memphis, Tennessee, is the only network member who is looking beyond clopidogrel and CYP2C19 under the auspices of the PGRN's Translational Pharmacogenetics Project. Since May, Relling and her colleagues have been genotyping youngsters for two genes, one involved in metabolizing cancer agents and the other linked to codeine response. "We're starting with the things that are home runs," she says. "In my opinion, these are the same genes that should be typed in every individual in the world."

Meanwhile, other centers not linked to the PGRN have even more ambitious programs. A pilot effort led by Howard McLeod at the University of North Carolina-Chapel Hill is considering how genetics affects about 40 different drug responses in people after they undergo solid organ transplants, and the University of Chicago's '1,200 Patients Project', slated to launch next year, will consider pharmacogenetic data when prescribing hundreds of different drugs to people with fairly broad eligibility criteria. "Our scope and our size are unique," says Peter O’Donnell, a medical oncologist at the University of Chicago. Since the information will be used to guide drug prescriptions that people would receive anyway, he says, "here's an area of genetics moving into clinical practice that we see as having little downside."

"I'm hopeful that it will become the norm," McLeod says of preemptive genotyping. "Certainly, patients deserve it."

Elie Dolgin 